\title{
HATE SPEECH IN SOCIAL MEDIA: PERCEPTIONS AND ATTITUDES OF HIGHER EDUCATION STUDENTS IN PORTUGAL
}

\author{
S. Santos, I. Amaral, R. Basílio Simões \\ University of Coimbra (PORTUGAL)
}

\begin{abstract}
Hate speech in social media has been an increasing concern, particularly during the last decade. Today, the rates of technology adoption are very high, particularly among the younger groups. Students are permanently connected through mobile technologies and the explosion of social media led to major changes in the flows of communication. Social platforms became the chosen vehicle for personal communication, for getting the news and for entertainment. At the same time, social media reflects the growing ideological polarization that is particularly visible on politics, religion, environmental and gender and sexuality issues. Hate speech is not a new phenomenon but it has specific characteristics in the online world that pose new challenges. International organizations have been promoting initiatives to support countries in tackling this issue. Social media platforms are also defining rules against diverse forms of intolerance particularly directed at vulnerable groups. Currently, there is a growing body of feminist research that has mainly focused on identifying how social media platforms can be toxic environments for women, namely by facilitating image-based sexual abuses and the sexual objectification of women and girls.

Higher education students are a critical group regarding this problem. Not only they are permanently connected, but they are also frequently less prepared to deal with disinformation and discriminatory digital interactions than they think. In this qualitative study, we want to know how higher education students in Portugal deal with online hate speech, particularly gender-focused hate speech. We present results from four focus groups that were conducted with both Portuguese and Brazilian students $(n=$ 28). Findings promote a deeper understanding of the proliferation of hate speech on social media, particularly towards female foreign students and point at the importance of promoting curricular and extra-curricular literacy programs, as well as mechanisms of monitoring on social platforms.
\end{abstract}

Keywords: Hate speech, Social media, Higher education students, Gender-focused hate speech.

\section{INTRODUCTION}

During the last 15 years, a decisive change occurred in young people's relationship with media and technology in developed societies. They live in permanent connectivity, like parts of a new collaborative culture [1], creating and spreading content through social platforms. This change has to be described beyond its technological dimension. The use of social media reflects new and wider media practices, social patterns and flows of information.

Despite the central role of knowledge in contemporary society, the quality of the information did not turn out to be a major concern for the general public. The last decade can be characterized by the tendency for information disorder [2] and infoxication [3], which are two elements that reflect the appearance of new producers of information - both individual and robotized [4], the degradation of facts as indisputable truths [5], and the growing crisis of journalism [6].

Information has become increasingly manipulated, and people are more exposed than ever to content that has other purposes beyond the promotion of social value. In Europe, especially since 2015, fake news became such a major concern, particularly for threatening democratic values. International organizations like the European Council and the European Commission, and companies like Facebook, Twitter or Google have been investing in tackling this issue.

The importance of building strong intellectual competencies has arisen as one of the main needs for living in a highly polarized era, particularly in a new informational ecosystem that has disinformation and manipulation as intrinsic parts [7]. This has become clearer, especially since Pennycook and Rand [8] found that "susceptibility to fake news is driven more by lazy thinking than it is by partisan bias".

It is precisely in this highly polarized world with considerable amounts of people that lack the competencies to deal with the particularities of communicating through the internet that hate speech has 
also became a major concern. As Keipi and colleagues [9] argue, "hate and aggression are potentially everywhere in human interaction, and that they are not necessarily limited to the most well-known examples such as racism and religious bigotry". Hence, hate was not born with the internet, but it is precisely the online environment, particularly social media, that has brought this topic to wider attention.

Both the openness and accessibility of the internet, which was seen right from the beginning as a deterritorialized forum for freedom are nuclear ideas to understand this problem. That is why freedom of speech is so frequently summoned to justify these kinds of behaviour. Nowadays, it also became easy to produce, spread content and to participate online, independently of people's ethical, moral or political intentions. In fact, cyberhate differs from offline situations precisely because it is associated with features like easy access, size of audience, anonymity, and instantaneousness [10]. Hate speech on the internet is a growing problem with political and social consequences. Online social networks are associated with the spreading of extremism, hate crime, terrorism and hate speech [11].

However, hate speech can assume many forms, some of which are even legal in diverse online contexts. This dynamic character makes it hard to find an all-embracing definition of hate speech. Cohen-Almagor describes it as "bias-motivated, hostile, malicious speech aimed at a person or a group of people because of some of their actual or perceived innate characteristics. It expresses discriminatory, intimidating, disapproving, antagonistic, and/or prejudicial attitudes toward those characteristics, which include gender, race, religion, ethnicity, color, national origin, disability, or sexual orientation. Hate speech is intended to injure, dehumanize, harass, intimidate, debase, degrade, and victimize the targeted groups, and to foment insensitivity and brutality against them" [12].

Diverse actions have been implemented during the last few years, especially on preventive and educational levels. Among other initiatives and institutions, the Council of Europe launched the "No Hate Speech Movement" in 2013, as a way to mobilize young people and fight the normalization of hostile speech: "the movement seeks to reduce the acceptance of hate speech both online and offline, through human rights education and awareness-raising, youth participation and media literacy" [13].

An institutional consensus about the importance of protecting human rights in this context has taken form. The Istanbul Convention is focused on the protection of women against violence, with particular attention to preventing domestic abuse. The Declaration of the Committee of Ministers of the Council of Europe recognizes the internet as a fertile space for radicalization and hate speech, emphasizing the importance of fighting stereotypes and promoting inclusive societies.

In Europe, the migration movements also led to waves of questioning identities, territorial limits and solidarity, giving rise to immense manifestations online [14]. Racist discourse also has risen on the internet, which led to the European Agency of Fundamental Rights to take actions to tackle the growing use of the internet for propaganda and hate speech [14].

The internet is not detached from the patriarchal structure of society. Hence, just like in the offline world, women are exposed to diverse forms of aggression, misogyny and anti-feminism online [15]. As the authors say, it is urgent to discuss and to act on this.

\section{METHODOLOGY}

For this study we conducted focus groups, which involve the interaction between participants as a source for research data. In social sciences and media studies, focused interviews with small homogeneous groups have proved to be useful to understand how participants interact, mobilise and contest media content while making sense of their everyday experiences ([16], [17], [18]). Twenty-eight Portuguese and Brazilian male and female higher education students took part in four focus groups conducted between October and December 2020. All the participants are users of social media, despite having diverse purposes and levels of use. We used topic guides and questions to structure and focus the discussions. The research was conducted to grasp the content of the ongoing discussion, but we also wanted to gain access to the dynamics of the interactions, in order to get insights into the participants' meanings and ways of understanding hate speech.

The sample consisted of 28 students from a Portuguese university in the academic year 2019/2020. The participants were 20 girls and 8 boys, aged between 18 and 25. Participants are Brazilian $(n=18)$ and Portuguese $(n=10)$. 


\section{RESULTS}

All the participants affirm that they access social media on a daily basis. The most used platforms are Instagram, Twitter, Facebook and YouTube. WhatsApp app is also very popular, especially among Brazilian students. Snapchat is common within Portuguese students.

Most students indicate the use of Facebook as being more restricted to private groups for sharing information. Participants also report to follow pages, search for news and connect with friends.

Instagram is used to follow people with common interests and to share photos and videos with friends. Students relate following friends and celebrities. Travel, entertainment and artistic photography are indicated as the topics that mostly appeal to students on Instagram.

Twitter is used to access news, interact with others, consume entertainment, and debate. YouTube is referred to as a medium for students to access entertainment, opinion and information content.

WhatsApp and Snapchat are used for conversation and interaction with others. A common aspect for all Brazilian students is to participate in family groups on WhatsApp. Portuguese students do not refer to family connections through these services.

The results show that students identify hate speech as offences and attacks. In a context where fake news and political polarization are always present, participants consider that the Internet has amplified hate speech and, to some extent, normalized it. Digital toxicity and aggression are directly related to the fact that the screen mediates hate speech (Table 1).

Table 1. Hate Speech Perceptions on Social Media.

M - Do you realize that social media platforms are also used to offend people?

M1 (FG1) - Yes, yes. There is something that is a kind of domino. Person A says something offensive about Person B. Person C supports Person A, but so fervently that it offends Person B. And so on. I think it happens a lot on social media (...), no one is expecting to offend, but I can say something that to me is basic and harmless, although there is someone in the world who sees that and get offended.

F1 (FG1) - Yeah, that's it. Well, we have to talk about what is an offence and the limits of offence.

M1 (FG1) - You see a text on the screen. It's not a person; it's just a text. And you feel more comfortable saying what you want. Anger and frustration flow much faster.

F1 (FG1) - People hide behind the screen. Without showing their faces, they can say whatever they want. Call people whatever they want.

M2 (FG1) - It is easy to think that there is a lot of hate speech on social media because watching the conservative and reactionary wave that crosses the world, especially the West ... We know that one of the biggest tools for affirming conservative ideas is the creation of profiles and bots to publish hate content on media pages, personalities, politicians.

The structure of patriarchal society is presented by students as the basis for political bias, given that it is based on male toxic speeches that are standardized and followed. Examples of Brazilian and US elections are retrieved by participants to validate their opinions on normalizing hate speech, whether online or offline. Participants identify different types of hate speech on social media such as racism, homophobia, religious intolerance, transphobia, xenophobia, male chauvinism, harassment (Table 2).

Table 2. Types of Hate Speech on Social Media.

$M$ - What types of hate speech do you encounter most on social media?

M1 (FG3) - In my case, the ones I see the most are racism and homophobia because there is so much on Twitter. And also because of the political issue in Brazil.

F1 (FG3) - Yes and the religious question as well.

M2 (FG3) - Transphobia also exists. And it is very problematic.

M3 (FG3) - I have personal contact with xenophobia.

$(\ldots)$ 
F2 (FG3) - With me, xenophobia is always allied with male chauvinism. I feel some attack because I'm a woman and a Brazilian. It happens on the street and social media. But I feel much stronger when it is in person, in the online networks I ignore.

$M$ - When you are the target of these situations, what do you do?

F2 (FG3) - I cry.

F1 (FG3) - I prefer to get out of the situation. I get away from the person, and I look for someone to talk, a friend or someone.

One of the types of violence that is most identified by the focus groups participants was online harassment of women (Table 3). The most common practices are comments, private messaging, sexting, sexually explicit content, and video viewing group invitations. One thing to note is the identification of men as the greatest aggressors, with older men being referred to more often by students. Participants also noted that anonymity encourages more aggressive attitudes by the offenders.

Table 3. Women's Online Harassment.

$M$ - Do you think there are platforms where hate speech is most visible?

F1 (FG4) - Harassment exists a lot on Instagram, but the platform has several ways to avoid this. Since you can't avoid the person causing it, we do what we can. For example, I always had my Instagram account open, and older men started following me and commenting on my photos. I was so uncomfortable with the situation that I preferred to put my Instagram account as private.

(...)

F2 (FG4) - On Facebook, some communities are closed in groups. People get in a bubble, they don't leave, and they normalize certain comments and thoughts.

F3 (FG4) - I've seen a lot of harassment on Twitter, especially with underage girls. 14- and 15-yearold girls posting photos on Twitter and lots of comments from older people like 'wow, too bad I can go to jail'. That kind of comments for younger girls. It is quite common.

F4 (FG4) - On Instagram, I am now receiving invitations from women who make viewing groups and offer sex. 'Do you wanna have sex?' 'Do you want some hot pictures?'. As it is an invitation, the person can accept or cancel. I always cancel, but I have a friend who recently, out of nowhere, received a picture of a man's genital organ.

The results show that body shaming focused on female bodies is another type of hate speech. Women's objectification is attributed to men. At the same time, women's comments are offensive to other women, focusing on their bodies. Participants consider that exposure of the body is natural and argue that the acceptance of bodies breaks beauty stereotypes (Table 4).

Table 4. Body Shaming.

F1 (FG2) - We see in the comments of men that what they do is objectify the woman. And women comment to diminish the woman, trying to make her feel bad for exposing herself. Maybe out of envy, I don't know. But I know this exists.

F2 (GF2) - And even by embarrassment when someone can't understand, for example, cellulite... they don't understand why we can accept this and why someone would publish a photo with cellulite because most people disguises and hides. These people get offended when others don't do the same.

$M$ - Does this has to do with changes in how intimacy is exposed today?

F2 (GF2) - I think there was always this idea. I remember my grandmother and friends. If a friend does something different, it's a scandal. They talk to each other, they criticize. It's basically the same thing only instead of doing it in secret with others, it's through a screen in someone's photo or someone's post, or in a comment.

M1 (FG2) - Sometimes even in private message.

F2 (GF2) - Exactly. Sometimes even in private message.

M2 (FG2) - Basically, online social networks only served as a megaphone for things that were already happening. 
F1 (FG2) - I think there has always been this kind of thought.

F3 (FG2) - Yes, just as there is more freedom for hate speech, so there is more freedom for people to start expressing more.

F2 (FG2) - In the movements more related to the acceptance of the body, they eventually adopted a shock strategy. Shocking attention-grabbing therapy for people to notice that they are doing this and this is really acceptable, and so I can do it too. It was this shock therapy that caused so much drama.

M1 (GF2) - But the truth is that it starts to work. People start to find it more normal. And that's how it should be. They are starting to find it more normal to have chubby models and thinner models. There is no longer that model idea that has to be extremely thin.

The results also show that young people consider some exposure of their intimacy to be reasonable. Amplification and anonymity are distinctive aspects regarding the online environment.

\section{CONCLUSIONS}

Development of ICT contributed to the transfer of bullying and gender-based violence to the virtual world among young people and against young people. This phenomenon is associated with intentional psychological violence, which involves negative behaviours such as lies, threats, insults, defamation, intimidation, rumours, provocation and social exclusion, among other attitudes.

Under the umbrella of media literacy, school dynamics should teach about gender violence and discrimination, raise awareness about the importance of media and digital literacy, acknowledge about the digital footprint, and be an active agent in promoting information in rights and risks on the Internet.

This ongoing study contributes to a deeper understanding of online gender hate and harassment. In this specific context, online gender-based violence is an overt expression of the discrimination, harassment and inequality that exists offline. However, anonymity and amplification are clearly presented as distinctive characteristics that pose new threats and challenges. The personal experiences of these female higher education students reinforce the need for actions that may lead to behavioural changes that reflect values such as equality, tolerance and respect.

\section{REFERENCES}

[1] H. Jenkins, S. Ford, J. Green, Spreadable media: Creating value and meaning in a networked culture. New York: New York University Press, 2013.

[2] C. Wardle, H. Derakhshan, "Information disorder. Toward an interdisciplinary framework for research and policymaking," Council of Europe Report, 27, 2017. Retrieved from https://rm.coe.int/information-disorder-toward-an-interdisciplinary-framework-forresearc/168076277c.

[3] L. M. Romero-Rodríguez, P. Casas-Moreno, M. C. Caldeiro-Pedreira, (2018). "Desinformación e info-xicación en las cuartas pantallas" in Competencia mediática en medios digitales emergentes (Eds I. Aguaded, L. M. Romero-Rodríguez), pp.73-92, Salamanca: Comunicación Social Ediciones y Publicaciones, 2018.

[4] S. Kumar, N. Shah, N., "False information on web and social media: A survey," arXiv preprint arXiv:1804.08559, 2018.

[5] P. Mihailidis, S. Viotty, "Spreadable spectacle in digital culture: Civic expression, fake news, and the role of media literacies in 'post-fact' society"," American Behavioral Scientist, vol. 61, no. 4, pp.441-454, 2017.

[6] J. Fuller, What is happening to news: The information explosion and the crisis in journalism. Chicago: The Chicago University Press, 2010.

[7] J. Figueira, S. Santos, "Percepción de las noticias falsas en universitarios de Portugal: análisis de su consumo y actitudes," El profesional de la información, vol. 28, no. 3, 2019.

[8] G. Pennycook, D. G. Rand, "Lazy, not biased: Susceptibility to partisan fake news is better explained by lack of reasoning than by motivated reasoning," Cognition, vol. 188, pp.39-50, 2018. 
[9] T. Keipi, M. Näisi, A. Oksanen, P. Räsänen, Online hate and harmful content. Cross-national perspectives. London: Routledge, 2017.

[10] A. Brown, "What is so special about online (as compared to offline) hate speech?," Ethnicities, vol. 18, no. 3, pp.297-326, 2018.

[11] N. Chetty, S. Alathur, "Hate speech review in the context of online social networks," Aggression and violent behavior, vol. 40, pp.108-118, 2018.

[12] R. Cohen-Almagor, "Fighting Hate and Bigotry on the Internet," Policy \& Internet, vol. 3, no. 3, pp.126, 2011.

[13] Council of Europe, "No Hate Speech Movement," 2013. Retrieved from https://www.coe.int/en/web/no-hate-campaign/no-hate-speech-movement

[14] S. Assimakopoulos, F. H. Baider, S. Millar, Online hate speech in the European Union: A discourseanalytic perspective. Heidelberg: Springer, 2017.

[15] D. Ging, E. Siapera, Gender-hate online. Understanding the new anti-feminism. Cham: Palgrave Macmillan, 2019.

[16] J. Kitzinger, "The methodology of focus groups: the importance of interaction between research participants," Sociology of Health and Illness, vol. 16, pp.103-121, 1994.

[17] D. L. Morgan, "Focus Groups and Social Interaction" in The SAGE Handbook of Interview Research (Eds. J. F. Gubrium, J. A. Holstein, A. B. Marvasti, K. D. McKinney), pp.161-176, Los Angeles, London, New Delhi, Singapore, Washington, DC: Sage, 2012.

[18] R. B. Simões, A. T. Peixinho, "Narratives as prison 'escapes': power, interaction and the coconstruction of the female prisoner by incarcerated women" in Narratives and social memory: theoretical and methodological approaches (Eds. R. Cabecinhas, L. Abadia), pp. 283-297. Braga: Communication and Society Research Centre, 2013. 